\title{
Dirac Quantization of Restricted QCD
}

\author{
Y.M. Cho ${ }^{1, a}$, Soon-Tae Hong ${ }^{2, b}$, J.H. Kim ${ }^{1}$, and Young-Jai Park ${ }^{3, c}$ \\ ${ }^{1}$ Center for Theoretical Physics and School of Physics \\ College of Natural Sciences, Seoul National University, Seoul 151-742, Korea \\ ${ }^{2}$ Department of Science Education and Research Institute for Basic Sciences \\ Ewha Womans University, Seoul 120-750 Korea \\ ${ }^{3}$ Department of Physics and Basic Science Research Institute, \\ Sogang University, C.P.O. Box 1142, Seoul 100-611, Korea
}

October 9, 2018

\begin{abstract}
We discuss the quantization of the restricted gauge theory of SU(2) QCD regarding it as a second-class constraint system, and construct the BRST symmetry of the constrained system in the framework of the improved Dirac quantization scheme. Our analysis tells that one could quantize the restricted QCD as if it is a first-class constraint system.
\end{abstract}

PACS: 11.10.-Z, 11.10.Ef, 11.30.-j, 12.38.-t

Keywords: Restricted QCD, improved Dirac quantization

aymcho@yongmin.snu.ac.kr; ${ }^{b}$ soonhong@ewha.ac.kr; ${ }^{c}$ yjpark@ccs.sogang.ac.kr 


\section{Introduction}

Understanding QCD and its confinement mechanism is probably one of the most fundamental problems in theoretical physics. It has long been speculated that the confinement could be explained by the condensation of the nonAbelian monopoles which could trigger the dual Meissner effect [1, 2, 3, 4]. In particular, it has been argued that the dual dynamics of the restricted QCD, restricted by the "Abelian projection" of the QCD potential, plays the important role in the confinement of color $[2,3,5]$. But understanding the dual dynamics of the restricted QCD which describes the interaction between the chromoelectric gluon and the chromomagnetic monopole has been non-trivial, because it describes the dynamics of a constrained system. The purpose of this paper is to clarify the dual dynamics and quantize the restricted QCD using the improved Dirac quantization method $[6,7,8]$.

The proof of the monopole condensation in QCD has been extremely difficult to achieve. The earlier attempts to prove the magnetic condensation with the effective action of QCD has failed because the external magnetic background is unstable due to the tachyonic modes $[9,10]$. Recently, however, there has been interesting new developments on this old problem. First, it has been shown that the instability of the magnetic background follows from the fact that the external background is not gauge invariant. Indeed, if we separate the magnetic background gauge independently from the quantum fluctuation of gluon and impose the gauge invariance to the magnetic background or the causality to the gluon fluctuation around the magnetic background, we can show from the resulting effective action of QCD that the magnetic background becomes a stable vacuum of QCD [11, 12].

Independent of this it has been conjectured that the Skyrme-Faddeev Lagrangian which allows knot-like topological solitons could be interpreted as an effective Lagrangian of QCD in the low energy limit [13, 14]. Although this conjecture may not be completely correct, it does reveal an interesting connection between the Skyrme-Faddeev theory and QCD, at least in some approximation $[15,16]$. The two theories have almost identical topological structures. Both admit the $\mathrm{Wu}$-Yang monopole characterized by the mapping $\pi_{2}\left(S^{2}\right)$ as a fundamental object, and allow the knot configurations classified by the mapping $\pi_{3}\left(S^{3}\right) \simeq \pi_{3}\left(S^{2}\right)$ as classical solutions [17, 18]. Besides, the knot of Skyrme-Faddeev theory can be mapped to QCD and interpreted to describe the multiple vacua of QCD [19]. Moreover, both can be interpreted 
as theories of confinement. The Skyrme-Faddeev theory screens (confines) the magnetic flux of the monopole-antimonopole pairs, while QCD confines the chromoelectric flux of the gluon-antigluon pairs. Furthermore, one can show that one may actually derive a generalized Skyrme-Faddeev action from the QCD effective action itself [16].

The crux behind these new developments is the so-called "Cho decomposition", the gauge independent decomposition of the non-Abelian potential into the restricted potential and the valence potential $[2,4]$. The virtue of the decomposition is that it clarifies the topological structure of the nonAbelian gauge theory, and naturally takes care of the topological characters in the dynamics. It has been well-known that the non-Abelian gauge theory has rich topological structures manifested by the non-Abelian monopoles $[20,21]$, the multiple vacua and the instantons $[22,23]$. One has to make sure that these topological characters are properly taken into account in the non-Abelian dynamics. In the specific case of $\mathrm{SU}(2)$, the relevant topology is the $\pi_{2}\left(S^{2}\right)$ which describes the non-Abelian monopoles, and the $\pi_{3}\left(S^{3}\right)$ which describes the multiple vacua and the corresponding vacuum tunneling by instantons. Since the decomposition of the non-Abelian connection contains these topological degrees explicitly, it can naturally take care of them in the non-Abelian dynamics [2, 4].

An important consequence of the decomposition is that it allows us to view QCD as the restricted gauge theory (made of the restricted potential) which is coupled to a gauge-covariant colored vector field (the valence potential). The restricted potential is defined in such a way that it allows a covariantly constant unit isovector $\hat{n}$ everywhere in space-time, which enables us to define the gauge-independent color direction everywhere in space-time and at the same time allows us to define the magnetic potential of the non-Abelian monopoles. Furthermore it has the full SU(2) gauge degrees of freedom, in spite of the fact that it is restricted $[2,4]$. Consequently the restricted QCD made of the restricted potential describes a very interesting dual dynamics of its own, and plays a crucial role in our understanding of QCD [5, 16].

On the other hand the restricted QCD is a constrained system, due to the presence of the topological field $\hat{n}$ which is constrained to have the unit norm. Physical system with constraints was systematically studied by Dirac [6], who showed that in the second-class constraint system one needs to use the Dirac brackets instead of the Poisson brackets to quantize the system. The standard Dirac method was later improved to convert the second-class constraints into 
first-class ones with the introduction of Stückelberg fields [7, 8]. Recently this improved Dirac quantization scheme has been applied to several areas of current interests such as the soliton models [24, 25, 26], high dense matter physics [27], and D-brane systems [28].

In this paper we discuss the second-class constraints of the restricted QCD and quantize the theory in the framework of the improved Dirac quantization method. In specific, we first quantize the constrained system and obtain the first-class effective Lagrangian, and discuss the Becci-Rouet-Stora-Tyutin (BRST) symmetry arising from the first-class effective Lagrangian. Our analysis tells that the restricted QCD, even though it is a second-class constraint system, could actually be quantized as a first-class constraint system.

The paper is organized as follows. In Section 2, we briefly recapitulate the restricted QCD. In Section 3, we analyze the constraint structure of the theory. In Section 4, we extend this theory to the first-class Hamiltonian system using the improved Dirac quantization scheme and obtain the firstclass Lagrangian using the Faddeev-Senjanovic formula. In Section 5, we construct the BRST symmetry of the restricted QCD after obtaining the first-class effective Lagrangian. Finally in Section 6 we discuss the physical implications of our work.

\section{Restricted QCD}

In this section we briefly review the restricted QCD. Consider $\mathrm{SU}(2)$ gauge theory for simplicity. A natural way to accommodate the topological degrees into the theory is to introduce a topological field $\hat{n}$ of unit length, and to decompose the connection into the Abelian projection part which leaves $\hat{n}$ a covariant constant and the remaining part which forms a covariant vector field $[2,4]$,

$$
\begin{gathered}
\vec{A}_{\mu}=A_{\mu} \hat{n}-\frac{1}{g} \hat{n} \times \partial_{\mu} \hat{n}+\vec{X}_{\mu}=\hat{A}_{\mu}+\vec{X}_{\mu}, \\
A_{\mu}=\hat{n} \cdot \vec{A}_{\mu}, \quad \hat{n}^{2}=1,
\end{gathered}
$$

where $A_{\mu}$ is the "electric" potential. Notice that the Abelian projection $\hat{A}_{\mu}$ is precisely the connection which leaves $\hat{n}$ invariant under the parallel transport and makes $\hat{n}$ a covariant constant,

$$
\hat{D}_{\mu} \hat{n}=\partial_{\mu} \hat{n}+g \hat{A}_{\mu} \times \hat{n}=0 .
$$


Under the infinitesimal gauge transformation

$$
\delta \hat{n}=-\vec{\alpha} \times \hat{n}, \quad \delta \vec{A}_{\mu}=\frac{1}{g} D_{\mu} \vec{\alpha}
$$

one has

$$
\delta A_{\mu}=\frac{1}{g} \hat{n} \cdot \partial_{\mu} \vec{\alpha}, \quad \delta \hat{A}_{\mu}=\frac{1}{g} \hat{D}_{\mu} \vec{\alpha}, \quad \delta \vec{X}_{\mu}=-\vec{\alpha} \times \vec{X}_{\mu}
$$

This shows that $\hat{A}_{\mu}$ by itself describes an $\mathrm{SU}(2)$ connection which enjoys the full $\mathrm{SU}(2)$ gauge degrees of freedom.

We emphasize that the crucial element in our decomposition (2.1) is the restricted potential $\hat{A}_{\mu}$ defined by the Abelian projection. Once this part is identified, the expression follows immediately due to the fact that the connection space (the space of all gauge potentials) forms an affine space [2, 4]. Indeed the affine nature of the connection space guarantees that one can describe an arbitrary potential simply by adding a gauge-covariant piece $\vec{X}_{\mu}$ to the restricted potential. The decomposition (2.1), which has recently become known as the "Cho decomposition" [14] or the "Cho-Faddeev-Niemi decomposition" [15], was introduced long time ago in an attempt to demonstrate the monopole condensation in QCD $[2,4]$. However, only recently the importance of the decomposition in clarifying the non-Abelian dynamics has become appreciated by many authors $[14,15,29,30]$. Indeed this decomposition has played a crucial role for us to establish the "Abelian dominance" in Wilson loops in QCD [5], and to calculate the effective action of QCD to prove the monopole condensation $[11,12,30]$.

To understand the physical meaning of our decomposition notice that the restricted potential $\hat{A}_{\mu}$ actually has a dual structure. Indeed the field strength made of the restricted potential is decomposed as

$$
\begin{gathered}
\hat{F}_{\mu \nu}=\left(F_{\mu \nu}+H_{\mu \nu}\right) \hat{n} \\
F_{\mu \nu}=\partial_{\mu} A_{\nu}-\partial_{\nu} A_{\mu} \\
H_{\mu \nu}=-\frac{1}{g} \hat{n} \cdot\left(\partial_{\mu} \hat{n} \times \partial_{\nu} \hat{n}\right)=\partial_{\mu} \tilde{C}_{\nu}-\partial_{\nu} \tilde{C}_{\mu}
\end{gathered}
$$

where $\tilde{C}_{\mu}$ is the "magnetic" potential $[2,4]$. Notice that one can always introduce the magnetic potential (at least locally section-wise), since one has 
the following identity

$$
\partial_{\mu} \tilde{H}_{\mu \nu}=0, \quad \tilde{H}_{\mu \nu}=\frac{1}{2} \epsilon_{\mu \nu \rho \sigma} H_{\rho \sigma} .
$$

This allows us to identify the non-Abelian monopole potential by

$$
\vec{C}_{\mu}=-\frac{1}{g} \hat{n} \times \partial_{\mu} \hat{n}
$$

in terms of which the magnetic field is expressed as

$$
\vec{H}_{\mu \nu}=\partial_{\mu} \vec{C}_{\nu}-\partial_{\nu} \vec{C}_{\mu}+g \vec{C}_{\mu} \times \vec{C}_{\nu}=-\frac{1}{g} \partial_{\mu} \hat{n} \times \partial_{\nu} \hat{n}=H_{\mu \nu} \hat{n} .
$$

Another important feature of $\hat{A}_{\mu}$ is that, as an $\mathrm{SU}(2)$ potential, it retains the full topological characteristics of the original non-Abelian potential $[2,4]$. Clearly the isolated singularities of $\hat{n}$ defines $\pi_{2}\left(S^{2}\right)$ which describes the nonAbelian monopoles. Indeed $\hat{A}_{\mu}$ with $A_{\mu}=0$ and $\hat{n}=\hat{r}$ (or equivalently, $\vec{C}_{\mu}$ with $\hat{n}=\hat{r}$ ) describes precisely the Wu-Yang monopole [20, 21]. Besides, with the $S^{3}$ compactification of $R^{3}, \hat{n}$ characterizes the Hopf invariant $\pi_{3}\left(S^{2}\right) \simeq$ $\pi_{3}\left(S^{3}\right)$ which describes the topologically distinct vacua $[22,23]$. This tells that the restricted gauge theory made of $\hat{A}_{\mu}$ could describe the dual dynamics which should play an essential role in $\mathrm{SU}(2)$ QCD, which displays the full topological characters of the non-Abelian gauge theory $[5,16]$.

With the connection (2.1), we have

$$
\begin{gathered}
\vec{F}_{\mu \nu}=\left(F_{\mu \nu}+H_{\mu \nu}\right) \hat{n}+\hat{D}_{\mu} \vec{X}_{\nu}-\hat{D}_{\nu} \vec{X}_{\mu}+g \vec{X}_{\mu} \times \vec{X}_{\nu}, \\
\hat{n} \cdot \vec{X}_{\mu}=0, \quad \hat{n} \cdot \hat{D}_{\mu} \vec{X}_{\nu}=0,
\end{gathered}
$$

so that the Yang-Mills Lagrangian density is expressed as

$$
\begin{aligned}
\mathcal{L}=- & \frac{1}{4} \hat{F}_{\mu \nu}^{2}-\frac{1}{4}\left(\hat{D}_{\mu} \vec{X}_{\nu}-\hat{D}_{\nu} \vec{X}_{\mu}\right)^{2}-\frac{g}{2} \hat{F}_{\mu \nu} \cdot\left(\vec{X}_{\mu} \times \vec{X}_{\nu}\right) \\
& -\frac{g^{2}}{4}\left(\vec{X}_{\mu} \times \vec{X}_{\nu}\right)^{2}+\lambda\left(\hat{n}^{2}-1\right)+\lambda_{\mu} \hat{n} \cdot \vec{X}_{\mu},
\end{aligned}
$$

where $\lambda$ and $\lambda_{\mu}$ are the Lagrangian multipliers.

Now we consider the restricted gauge theory made of the Abelian projection without $\vec{X}_{\mu}$

$$
\mathcal{L}_{0}=-\frac{1}{4} \hat{F}_{\mu \nu}^{2}+\lambda\left(\hat{n}^{2}-1\right)
$$


As we have emphasized the theory has a full $\mathrm{SU}(2)$ gauge invariance. More importantly the restricted gauge theory describes the dual dynamics of QCD with the dynamical degrees of the maximal Abelian subgroup $\mathrm{U}(1)$ as the electric component and the topological degrees of $\mathrm{SU}(2)$ as the magnetic component $[2,4]$. In the conventional analysis the topological degrees have often been neglected because one could always make $\hat{n}$ trivial and remove it from the theory by a gauge transformation, at least sectionwise locally. However notice that, if one includes the topologically non-trivial sectors into the theory, one can not neglect $\hat{n}$ because it can not be removed by a smooth gauge transformation. In fact it becomes dynamical when one quantizes the theory $[5,16]$.

Obviously the restricted QCD is a constrained system, because the topological field $\hat{n}$ is constrained. So we have to decide how to deal with the constraint $\hat{n}^{2}=1$ when we quantize the theory. In general we could impose the constraint strictly throughout the quantization, requiring the quantum fluctuation to respect the constraint. Or we could relax the constraint, pretending that the quantum fluctuation need not respect the constraint. In this paper we take the second attitude, and quantize the restricted QCD treating it as a system with a second-class constraint. With this understanding we are ready to derive the first-class Hamiltonian for the restricted QCD in the framework of the improved Dirac quantization scheme [7, 8], and derive the first-class Hamiltonian for the restricted QCD.

\section{Constraints and Dirac commutators}

In this section, we apply the Dirac quantization scheme [6] to the restricted QCD with the Lagrangian (2.11),

$$
\begin{gathered}
L_{0}=\int \mathrm{d}^{3} x\left[-\frac{1}{4} \hat{F}_{\mu \nu}^{2}\right]=\int \mathrm{d}^{3} x\left[-\frac{1}{4} G_{\mu \nu}^{2}\right], \\
G_{\mu \nu}=F_{\mu \nu}+H_{\mu \nu},
\end{gathered}
$$

regarding it as a second-class constraint system with the constraint,

$$
\Omega_{1}=n^{a} n^{a}-1=\hat{n}^{2}-1 \approx 0
$$


By performing the Legendre transformation, one can obtain the momenta conjugate to the fields $A^{\mu}$

$$
\Omega_{1}^{A}=\Pi^{0}=0, \quad \Pi^{i}=G^{i o}
$$

to yield the canonical Hamiltonian,

$$
H=\int \mathrm{d}^{3} x\left[\frac{1}{2} \Pi_{i} \Pi_{i}+\frac{1}{4} G_{i j}^{2}-A_{0} \partial_{i} \Pi_{i}\right] .
$$

Notice that here one can explicitly include the constraint term $\lambda \Omega_{1}$ in the Hamiltonian (3.4) to yield the primary constraint $\Omega_{1}^{\lambda}=\pi_{\lambda}=0$ with the momentum $\pi_{\lambda}$ conjugate to $\lambda$ and, after its time evolution, the secondary constraint $\Omega_{2}^{\lambda}$. Since these constraints $\Omega_{i}^{\lambda}$ satisfy a trivial first-class algebra, we will treat the geometric constraint $\Omega_{1}$ as usual without explicit inclusion of the $\lambda \Omega_{1}$ term in the canonical Hamiltonian (3.4). With this understanding the time evolution of the constraint $\Omega_{1}^{A}$ with the above Hamiltonian yields an additional secondary constraint

$$
\Omega_{2}^{A}=\partial_{i} \Pi_{i}=0
$$

Note that, the above two constraints $\Omega_{k}^{A}$ are first-class which satisfy the constraint Lie algebra

$$
\left\{\Omega_{k}^{A}(x), \Omega_{k^{\prime}}^{A}(y)\right\}=0 .
$$

On the other hand, the time evolution of the geometrical constraint $\Omega_{1}$ with the Hamiltonian (3.4) yields

$$
\left\{\Omega_{1}, H\right\}=-\frac{2 g}{c} \hat{n} \cdot \hat{\pi}, \quad c=\frac{1}{g} \hat{n}^{2}\left(\partial_{i} \hat{n}\right)^{2},
$$

where $\hat{\pi}$ is the momenta conjugate to $\hat{n}$ given by

$$
\hat{\pi}=\frac{1}{g}\left(\hat{n} \times \partial_{i} \hat{n}\right) \Pi_{i} .
$$

The Poisson bracket (3.7) then yields another secondary constraint

$$
\Omega_{2}=\hat{n} \cdot \hat{\pi} \approx 0
$$


which, together with $\Omega_{1}$, forms a second-class constraint algebra

$$
\Delta_{k k^{\prime}}(x, y)=\left\{\Omega_{k}(x), \Omega_{k^{\prime}}(y)\right\}=2 \epsilon_{k k^{\prime}} \hat{n}^{2} \delta(x-y)
$$

with $\epsilon_{12}=-\epsilon_{21}=1$. Note that they are involutive with the above first-class constraints $\Omega_{k}^{A}$

$$
\left\{\Omega_{k}^{A}(x), \Omega_{k^{\prime}}(y)\right\}=0 .
$$

After some algebraic manipulation, we construct the Poisson bracket

$$
\begin{aligned}
\left\{n^{a}(x), n^{b}(y)\right\} & =\left\{\pi^{a}(x), \pi^{b}(y)\right\}=\left\{A_{\mu}(x), A_{\nu}(y)\right\}=\left\{\Pi_{\mu}(x), \Pi_{\nu}(y)\right\}=0, \\
\left\{n^{a}(x), \pi^{b}(y)\right\} & =\delta^{a b} \delta(x-y), \\
\left\{A_{\mu}(x), \Pi_{\nu}(y)\right\} & =g_{\mu \nu} \delta(x-y), \\
\left\{n^{a}(x), A_{\mu}(y)\right\} & =\left\{n^{a}(x), \Pi_{0}(y)\right\}=\left\{\pi^{a}(x), A_{0}(y)\right\}=\left\{\pi^{a}(x), \Pi_{0}(y)\right\}=0, \\
\left\{n^{a}(x), \Pi_{i}(y)\right\} & =\frac{1}{c} \epsilon^{a b c} n^{b} \partial_{i} n^{c} \delta(x-y), \\
\left\{\pi^{a}(x), A_{i}(y)\right\} & =\frac{1}{g} \epsilon^{a b c} n^{b} \partial_{i} n^{c} \delta(x-y), \\
\left\{\pi^{a}(x), \Pi_{i}(y)\right\} & =-\frac{2}{g c} n^{a}\left(\partial_{i} \hat{n} \cdot \partial_{j} \hat{n}\right) \Pi_{j} \delta(x-y)+\text { total derivative. }
\end{aligned}
$$

Here it is amusing to note that, even though the derivation of the secondary constraint (3.9) and the Poisson algebra (3.12) in our theory are quite nontrivial, the set of the constraints $\Omega_{i}$ are exactly the same as those of the $\mathrm{O}(3)$ nonlinear sigma model [25], where one can easily construct the constraints.

With the Poisson bracket we can now construct the Dirac brackets defined as

$$
\begin{gathered}
\{A(x), B(y)\}_{D}=\{A(x), B(y)\} \\
-\int d^{2} z d^{2} z^{\prime}\left\{A(x), \Omega_{k}(z)\right\} \Delta^{k k^{\prime}}\left\{\Omega_{k^{\prime}}\left(z^{\prime}\right), B(y)\right\},
\end{gathered}
$$

where $\Delta^{k k^{\prime}}$ is the inverse of $\Delta_{k k^{\prime}}$ defined by (3.10). With this we obtain the Dirac brackets

$$
\left\{n^{a}(x), \pi^{b}(y)\right\}_{D}=\left(\delta_{a b}-\frac{n^{a} n^{b}}{\hat{n}^{2}}\right) \delta(x-y),
$$




$$
\begin{aligned}
\left\{\pi^{a}(x), \pi^{b}(y)\right\}_{D} & =\frac{1}{\hat{n}^{2}}\left(n^{b} \pi^{a}-n^{a} \pi^{b}\right) \delta(x-y) \\
\left\{\pi^{a}(x), \Pi_{i}(y)\right\}_{D} & =-\frac{1}{g c} n^{a}\left(\partial_{i} \hat{n} \cdot \partial_{j} \hat{n}\right) \Pi_{j} \delta(x-y)+\text { total derivative } \\
\{A(x), B(y)\}_{D} & =\{A(x), B(y)\}, \quad \text { for other commutators. }
\end{aligned}
$$

Here note that the structure of the Dirac commutator $\left\{\pi^{a}(x), \Pi_{i}(y)\right\}_{D}$ is nontrivial as much as that of $\left\{\pi^{a}(x), \pi^{b}(y)\right\}_{D}$, since $\Pi_{i}$ is closely related to $\hat{\pi}$ as shown in Eq. (3.8).

\section{First-class Hamiltonian}

Following the improved Dirac quantization scheme [7, 8] which systematically converts the second-class constraints into the first-class ones, we introduce two Stückelberg fields $\left(\theta, \pi_{\theta}\right)$ with the Poisson brackets

$$
\left\{\theta(x), \pi_{\theta}(y)\right\}=\delta(x-y),
$$

to obtain the first-class constraints as follows

$$
\begin{gathered}
\tilde{\Omega}_{1}=\Omega_{1}+2 \theta, \\
\tilde{\Omega}_{2}=\Omega_{2}-\hat{n}^{2} \pi_{\theta},
\end{gathered}
$$

which yield a strongly involutive first-class Lie algebra

$$
\left\{\tilde{\Omega}_{k}(x), \tilde{\Omega}_{k^{\prime}}(y)\right\}=0 .
$$

Note that the physical fields $\hat{n}$ are geometrically constrained to fulfill the modified norm $\hat{n}^{2}=1-2 \theta$.

Now, we construct the first-class physical fields $\tilde{\mathcal{F}}=\left(\tilde{n}^{a}, \tilde{\pi}^{a}\right)$ corresponding to the original fields $\mathcal{F}=\left(n^{a}, \pi^{a}\right)$ demanding that they are strongly involutive,

$$
\left\{\tilde{\Omega}_{k}, \tilde{\mathcal{F}}\right\}=0
$$

After some algebra, we obtain the first-class physical fields as

$$
\tilde{n}^{a}=n^{a}\left(\frac{\hat{n}^{2}+2 \theta}{\hat{n}^{2}}\right)^{1 / 2}, \quad \tilde{\pi}^{a}=\left(\pi^{a}-n^{a} \pi_{\theta}\right)\left(\frac{\hat{n}^{2}}{\hat{n}^{2}+2 \theta}\right)^{1 / 2},
$$




$$
\begin{gathered}
\tilde{A}_{\mu}=A_{\mu}, \quad \tilde{\Pi}_{\mu}=\Pi_{\mu}, \\
\tilde{G}_{i j}=G_{i j}+H_{i j}\left[\left(\frac{\hat{n}^{2}+2 \theta}{\hat{n}^{2}}\right)^{3 / 2}-1\right], \quad \tilde{G}_{0 i}=G_{0 i} .
\end{gathered}
$$

Since any functional of the first-class fields $\tilde{\mathcal{F}}$ is also first-class [31], we easily construct a first-class Hamiltonian in terms of the above first-class physical variables omitting infinite iteration procedure to arrive at

$$
\tilde{H}=\int \mathrm{d}^{3} x\left[\frac{1}{2} \tilde{\Pi}_{i} \tilde{\Pi}_{i}+\frac{1}{4} \tilde{G}_{i j}^{2}-\tilde{A}_{0} \partial_{i} \tilde{\Pi}_{i}\right] .
$$

We then directly rewrite this Hamiltonian in terms of the original as well as Stückelberg fields to obtain

$$
\tilde{H}=\int \mathrm{d}^{3} x\left[\frac{1}{2} \Pi_{i} \Pi_{i}+\frac{1}{4}\left(G_{i j}+H_{i j} D\right)^{2}-A_{0} \partial_{i} \Pi_{i}\right],
$$

where $D=R^{3 / 2}-1$ with the rescaling factor $R$

$$
R=\frac{\hat{n}^{2}+2 \theta}{\hat{n}^{2}}
$$

Note that $\tilde{H}$ is strongly involutive with the first-class constraints,

$$
\left\{\tilde{\Omega}_{k}, \tilde{H}\right\}=0 .
$$

Next, we consider the partition function of the theory in order to present the Lagrangian corresponding to the first-class Hamiltonian $\tilde{H}$ in Eq.(4.4). The starting partition function in the phase space is then given by the Faddeev-Senjanovic formula [32] as follows

$$
\begin{aligned}
& Z=N \int \mathcal{D} n^{a} \mathcal{D} \pi^{a} \mathcal{D} A_{\mu} \mathcal{D} \Pi_{\mu} \mathcal{D} \theta \mathcal{D} \pi_{\theta} \prod_{i, j=1}^{2} \delta\left(\tilde{\Omega}_{i}\right) \delta\left(\Gamma_{j}\right) \\
& \cdot \operatorname{det}\left|\left\{\tilde{\Omega}_{i}, \Gamma_{j}\right\}\right| \exp \left[i \int \mathrm{d} t L\right] \\
& L=\int \mathrm{d}^{3} x\left[\pi^{a} \dot{n}^{a}+\Pi_{\mu} \dot{A}_{\mu}+\pi_{\theta} \dot{\theta}-\tilde{H}\right]
\end{aligned}
$$

where the gauge fixing conditions $\Gamma_{i}$ are chosen so that the determinant occurring in the functional measure is nonvanishing. 
Now, exponentiating the delta function $\delta\left(\tilde{\Omega}_{2}\right)$ with

$$
\delta\left(\tilde{\Omega}_{2}\right)=\int \mathcal{D} \xi \exp \left[i \int \mathrm{d} t \xi \tilde{\Omega}_{2}\right],
$$

and performing the integration over $\pi^{a}, \Pi_{\mu}, \pi_{\theta}$, and $\xi$, we obtain the following partition function and Lagrangian

$$
\begin{gathered}
Z=N \int \mathcal{D} n^{a} \mathcal{D} A_{\mu} \mathcal{D} \theta \delta\left(\tilde{\Omega}_{1}\right) \prod_{i=1}^{2} \delta\left(\Gamma_{i}\right) \operatorname{det}\left|\left\{\tilde{\Omega}_{i}, \Gamma_{j}\right\}\right| \exp \left[i \int \mathrm{d} t L\right], \\
L=L_{0}+L_{W Z}, \\
L_{W Z}=\int \mathrm{d}^{3} x\left[-\frac{1}{4} H_{i j}\left(2 G_{i j}+H_{i j} D\right) D\right],
\end{gathered}
$$

which is the desired first-class Lagrangian corresponding to the first-class Hamiltonian (4.4) and is invariant under the following gauge transformations

$$
\delta n^{a}=n^{a} \epsilon, \quad \delta \theta=-(1-2 \theta) \epsilon, \quad \delta A_{\mu}=0 .
$$

Here one notes that, by using the first-class observables $\tilde{G}_{\mu \nu}$ in Eq. (4.2), the Lagrangian (4.7) can be reshuffled to yield the Lorentz invariant form

$$
L=\int \mathrm{d}^{3} x\left[-\frac{1}{4} \tilde{G}_{\mu \nu}^{2}\right] .
$$

\section{BRST symmetry}

In this section, in order to obtain the effective Lagrangian, we introduce two canonical sets of ghosts and anti-ghosts together with auxiliary fields in the framework of the Batalin-Fradkin-Vilkovisky formalism [33, 34], which is applicable only to theories with the first-class constraints,

$$
\left(\mathcal{C}_{i}, \mathcal{P}_{i}^{\dagger}\right), \quad\left(\mathcal{P}_{i}, \mathcal{C}_{i}^{\dagger}\right), \quad\left(N_{i}, B_{i}\right), \quad(i=1,2)
$$

which satisfy the super-Poisson algebra

$$
\left\{\mathcal{C}_{i}(x), \mathcal{P}_{j}^{\dagger}(y)\right\}=\left\{\mathcal{P}_{i}(x), \mathcal{C}_{j}^{\dagger}(y)\right\}=\left\{N_{i}(x), B_{j}(y)\right\}=\delta_{i j} \delta(x-y),
$$

where the super-Poisson bracket is defined as

$$
\{A, B\}=\left.\left.\frac{\delta A}{\delta q}\right|_{r} \frac{\delta B}{\delta p}\right|_{l}-\left.\left.(-1)^{\eta_{A} \eta_{B}} \frac{\delta B}{\delta q}\right|_{r} \frac{\delta A}{\delta p}\right|_{l} .
$$


Here $\eta_{A}$ and $\eta_{B}$ denotes the number of fermions called ghost number in $A$ and $B$, and the subscript $r$ and $l$ imply right and left derivatives, respectively. In this formalism, the nilpotent BRST charge $Q$ and the fermionic gauge fixing function $\Psi$ are given as

$$
\begin{aligned}
& Q=\int \mathrm{d}^{3} x\left(\mathcal{C}_{i} \tilde{\Omega}_{i}+\mathcal{P}_{i} B_{i}\right), \\
& \Psi=\int \mathrm{d}^{3} x\left(\mathcal{C}_{i}^{\dagger} \chi_{i}+\mathcal{P}_{i}^{\dagger} N_{i}\right) .
\end{aligned}
$$

Now we choose a gauge

$$
\chi_{1}=\Omega_{1}, \quad \chi_{2}=\Omega_{2}
$$

With this the BRST charge $Q$ and the fermionic gauge fixing function $\Psi$ satisfy the following relations

$$
\{Q, \tilde{H}\}=0, \quad Q_{2}=\{Q, Q\}=0, \quad\{\{\Psi, Q\}, Q\}=0
$$

The quantum Lagrangian is then described by

$$
\begin{gathered}
L=\int \mathrm{d}^{3} x\left(\pi^{a} \dot{n}^{a}+\Pi_{\mu} \dot{A}_{\mu}+\pi_{\theta} \dot{\theta}+B_{2} \dot{N}_{2}+\mathcal{P}_{i}^{\dagger} \dot{\mathcal{C}}_{i}+\mathcal{C}_{2}^{\dagger} \dot{\mathcal{P}}_{2}\right)-H_{t o t}, \\
H_{t o t}=\tilde{H}-\{Q, \Psi\}
\end{gathered}
$$

where we have suppressed the term

$$
\int \mathrm{d}^{3} x\left(B_{1} \dot{N}_{1}+\mathcal{C}_{1}^{\dagger} \dot{\mathcal{P}}_{1}\right)=\left\{Q, \int \mathrm{d}^{3} x \mathcal{C}_{1}^{\dagger} \dot{N}_{1}\right\}
$$

by replacing $\chi_{1}$ with $\chi_{1}+\dot{N}_{1}$.

Integrating out $N_{1}, B_{1}, \mathcal{C}_{1}^{\dagger}, \mathcal{C}_{1}$, and the momentum fields by performing path integration in the BRST scheme, we can obtain the Lagrangian of the form

$$
L=\int \mathrm{d}^{3} x\left[-\frac{1}{4} G_{\mu \nu}^{2}-\frac{1}{4} H_{i j}\left(2 G_{i j}+H_{i j} D\right) D+B \dot{N}+\dot{\mathcal{C}}^{\dagger} \dot{\mathcal{C}}\right]
$$

where

$$
N=N_{2}, \quad B=B_{2}, \quad \mathcal{C}^{\dagger}=\mathcal{C}_{2}^{\dagger}, \quad \mathcal{C}=\mathcal{C}_{2}
$$


Identifying

$$
N=-B+\frac{\dot{\theta}}{1-2 \theta}
$$

we obtain

$$
\begin{gathered}
L=L_{0}+L_{W Z}+L_{g h}, \\
L_{g h}=\int \mathrm{d}^{3} x\left[-\frac{\partial_{\mu} B \partial^{\mu} \theta}{1-2 \theta}+\partial_{\mu} \mathcal{C}^{\dagger} \partial^{\mu} \mathcal{C}\right]
\end{gathered}
$$

which is invariant under the BRST transformation

$$
\begin{gathered}
\delta_{B} n^{a}=\lambda n^{a} \mathcal{C}, \quad \delta_{B} \theta=-\lambda(1-2 \theta) \mathcal{C}, \quad \delta_{B} A_{\mu}=0 \\
\delta_{B} \mathcal{C}^{\dagger}=-\lambda B, \quad \delta_{B} \mathcal{C}=0, \quad \delta_{B} B=0 .
\end{gathered}
$$

Now, using the first-class observables $\tilde{G}_{\mu \nu}$ in Eq. (4.2), we can obtain the Lorentz invariant form of the Lagrangian (5.7) as follows

$$
L=\int \mathrm{d}^{3} x\left[-\frac{1}{4} \tilde{G}_{\mu \nu}^{2}-\frac{\partial_{\mu} B \partial_{\mu} \theta}{1-2 \theta}+\partial_{\mu} \mathcal{C}^{\dagger} \partial_{\mu} \mathcal{C}\right] .
$$

Moreover, the above Lagrangian can be rewritten in terms of the first-class physical fields

$$
\begin{gathered}
Z=N \int \mathcal{D} \tilde{n}^{a} \mathcal{D} \tilde{A}_{\mu} \mathcal{D} \tilde{\theta} \mathcal{D} B \mathcal{D} \mathcal{C} \mathcal{D} \mathcal{C}^{\dagger} \delta\left(\tilde{\Omega}_{1}\right) \exp \left[i \int \mathrm{d} t L\right] \\
L=\int \mathrm{d}^{3} x\left[-\frac{1}{4} \tilde{G}_{\mu \nu}^{2}+\frac{1}{3} \partial_{\mu} B \partial_{\mu}(\ln \tilde{\theta})+\partial_{\mu} \mathcal{C}^{\dagger} \partial_{\mu} \mathcal{C}\right]
\end{gathered}
$$

where

$$
\tilde{\theta}=(1-2 \theta)^{3 / 2}
$$

Note that in this form of Lagrangian (5.10) the BRST transformation (5.8) is expressed by

$$
\begin{aligned}
& \delta_{B} \tilde{n}^{a}=0, \quad \delta_{B} \tilde{\theta}=3 \lambda \tilde{\theta} \mathcal{C}, \delta_{B} \tilde{A}_{\mu}=0 \\
& \delta_{B} \mathcal{C}^{\dagger}=-\lambda B, \quad \delta_{B} \mathcal{C}=0, \quad \delta_{B} B=0 .
\end{aligned}
$$


With the above discussion we notice that the gauge condition (5.2) requires $\theta=0$, and effectively removes it from the Lagrangian (5.9). In this case $\tilde{A}_{\mu}, \tilde{n}$, and $\tilde{G}_{\mu \nu}$ becomes identical to $\hat{A}_{\mu}, \hat{n}$, and $G_{\mu \nu}$. Furthermore the ghost fields $\mathcal{C}$ and $\mathcal{C}^{\dagger}$ completely decouple from the gauge field $\hat{A}_{\mu}$. So we finally have

$$
\begin{gathered}
Z=N \int \mathcal{D} n^{a} \mathcal{D} A_{\mu} \delta\left(\hat{n}^{2}-1\right) \exp \left[i \int \mathrm{d} t L\right], \\
L=\int \mathrm{d}^{3} x\left[-\frac{1}{4} \hat{F}_{\mu \nu}^{2}\right],
\end{gathered}
$$

This shows that the improved Dirac quantization rule and the direct quantization with the constraint $\hat{n}^{2}=1$ produce an identical result, at least in the gauge (5.2). An interesting problem is to prove the equivalence of the two approaches in an arbitrary gauge.

It should be remarked that, in order to complete the quantization of the restricted QCD described by the above Lagrangian (5.12), we still have to fix the gauge degrees of $\mathrm{SU}(2)$ even after the gauge fixing (5.2). For example we may choose the Lorentz gauge as the $\mathrm{SU}(2)$ gauge fixing condition [16]

$$
\partial_{\mu} \hat{A}_{\mu}=0 \text {, }
$$

which can be splitted into two parts

$$
\begin{gathered}
\partial_{\mu} A_{\mu}=0, \\
\hat{n} \times \partial^{2} \hat{n}-g A_{\mu} \partial_{\mu} \hat{n}=0 .
\end{gathered}
$$

In this case the corresponding Faddeev-Popov determinant is given by

$$
M_{a b}=\frac{\delta\left(\partial_{\mu} \hat{A}_{\mu}\right)_{a}}{\delta \alpha^{b}}=\left(\partial_{\mu} \hat{D}_{\mu}\right)_{a b}
$$

from which we obtain

$$
\begin{gathered}
Z=N \int \mathcal{D} n^{a} \mathcal{D} A_{\mu} \mathcal{D} \vec{c}^{\dagger} \mathcal{D} \vec{c} \delta\left(\hat{n}^{2}-1\right) \exp \left[i \int \mathrm{d} t L_{e f f}\right], \\
L_{e f f}=\int \mathrm{d}^{3} x\left[-\frac{1}{4} \hat{F}_{\mu \nu}^{2}\right. \\
\left.-\frac{1}{2 \xi}\left(\left(\partial_{\mu} A_{\mu}\right)^{2}+\left(\hat{n} \times \partial^{2} \hat{n}-g A_{\mu} \partial_{\mu} \hat{n}\right)^{2}\right)+\vec{c}^{\dagger} \cdot \partial_{\mu} \hat{D}_{\mu} \vec{c}\right],
\end{gathered}
$$

where $\vec{c}$ and $\vec{c}^{\dagger}$ are the ghost fields associated with the determinant (5.15) [16]. 


\section{Conclusions}

In this paper, we have discussed the restricted QCD, which possesses the second-class constraints, to construct the first class Hamiltonian and firstclass effective Lagrangian in the framework of the improved Dirac quantization scheme. Furthermore, with the first-class effective Lagrangian, we have constructed the BRST symmetry of the restricted QCD.

The lesson that we learn from the discussion is that the improved Dirac quantization method guarantees that, in the restricted QCD, the second-class constraint (3.2) can be treated as if it is a first-class constraint. This tells that we no longer have to worry about whether the constraint of the topological field $\hat{n}$ is of the first-class or second-class, when we quantize the restricted QCD. This is remarkable. This has been demonstrated by a proper choice of the gauge (5.2). The remaining problem is to establish the equivalence of the two approaches in an arbitrary gauge where $\theta$ is not zero, which is worth further investigation.

Two of the authors (YMC and STH) would like to thank the hospitality of J. Michelsson and E. Langman of Department of Theoretical Physics at Royal Institute of Technology and A. Niemi of Department of Theoretical Physics at Uppsala University, where this work has been initiated. The work of YMC and JHK is supported in part by the ABRL Project of Korea Research Foundation (KRF-R14-2003-012-01002-0), and the work of STH

and YJP was supported by the Korea Research Foundation Grant KRF-2006331-C00071 and KRF-2005-015-C00105 funded by Korean Government.

\section{References}

[1] Y. Nambu, Phys. Rev. D 10, 4262 (1974); S. Mandelstam, Phys. Rep. 23, 245 (1976); A. Polyakov, Nucl. Phys. B 120, 429 (1977).

[2] Y.M. Cho, Phys. Rev. D 21, 1080 (1980); J. Korean Phys. Soc. 17, 266 (1984).

[3] G. 't Hooft, Nucl. Phys. B 190, 455 (1981). 
[4] Y.M. Cho, Phys. Rev. Lett. 46, 302 (1981); Phys. Rev. D 23, 2415 (1981).

[5] Y.M. Cho, Phys. Rev. D 62, 074009 (2000).

[6] P.A.M. Dirac, Lectures in Quantum Mechanics (Yeshiva University, New York, 1964).

[7] I.A. Batalin and E.S. Fradkin, Phys. Lett. B 180, 157 (1986); Nucl. Phys. B 279, 514 (1987); I.A. Batalin and I.V. Tyutin, Int. J. Mod. Phys. A 6, 3255 (1991).

[8] S.T. Hong and Y.J. Park, Phys. Rep. 358, 143 (2002).

[9] G. K. Savvidy, Phys. Lett. B 71, 133 (1977); N. K. Nielsen and P. Olesen, Nucl. Phys. B 144, 485 (1978).

[10] A. Yildiz and P. Cox, Phys. Rev. D 21, 1095 (1980); M. Claudson, A. Yilditz, and P. Cox, Phys. Rev. D 22, 2022 (1980).

[11] Y.M. Cho, H.W. Lee, and D.G. Pak, Phys. Lett. B 525, 347 (2002); Y.M. Cho and D.G. Pak, Phys. Rev. D 65, 074027 (2002).

[12] Y.M. Cho, D.G. Pak, and M. Walker, JHEP 05, 073 (2004); Y.M. Cho and M.L. Walker, Mod. Phys. Lett. A 19, 2707 (2004); Y.M. Cho, J.H. Kim, and D.G. Pak, Mod. Phys. Lett. A 21, in press.

[13] L. Faddeev and A. Niemi, Nature 387, 58 (1997); R. Battye and P. Sutcliffe, Phys. Rev. Lett. 81, 4798 (1998).

[14] L. Faddeev and A. Niemi, Phys. Rev. Lett. 82, 1624 (1999); Phys. Lett. B 449, 214 (1999).

[15] E. Langman and A. Niemi, Phys. Lett. B 463, 252 (1999); S. Shabanov, Phys. Lett. B 458, 322 (1999); B 463, 263 (1999); H. Gies, Phys. Rev. D 63, 125023 (2001).

[16] W.S. Bae, Y.M. Cho, and S.W. Kimm, Phys. Rev. D 65, 025005 (2001).

[17] Y.M. Cho, Phys. Rev. Lett. 87, 252001 (2001); Y.M. Cho, Phys. Lett. B 603, 88 (2004). 
[18] Y.M. Cho, Phys. Lett. B 616, 101 (2005).

[19] P. van Baal and A. Wipf, Phys. Lett. B 515, 181 (2001); Y.M. Cho, hep-th/0409246, Phys. Lett. B, in press.

[20] T.T. Wu and C.N. Yang, Phys. Rev. D 12, 3845 (1975).

[21] Y.M. Cho, Phys. Rev. Lett. 44, 1115 (1980); Phys. Lett. B 115, 125 (1982); Y.D. Kim, I.G. Koh, and Y.J. Park, Phys. Rev. D 25, 587 (1982); W.S. l'Yi, Y.J. Park, I.G. Koh, and Y. Kim, Phys. Rev. Lett. 49, 1229 (1982).

[22] A. Belavin, A. Polyakov, A. Schwartz, and Y. Tyupkin, Phys. Lett. B 59, 85 (1975); G. 't Hooft, Phys. Rev. Lett. 37, 8 (1976).

[23] Y.M. Cho, Phys. Lett. B 81, 25 (1979).

[24] W. Oliveira and J.A. Neto, Int. J. Mod. Phys. A 12, 4895 (1997); S.T. Hong, Y.W. Kim, and Y.J. Park, Phys. Rev. D 59, 114026 (1999); S.T. Hong and Y.J. Park, Phys. Rev. D 63, 054018 (2001).

[25] S.T. Hong, W.T. Kim, and Y.J. Park, Phys. Rev. D 60, 125005 (1999).

[26] S.T. Hong and A.J. Niemi, Phys. Rev. D 72, 127701 (2005).

[27] D.K. Hong, S.T. Hong, and Y.J. Park, Phys. Lett. B 499, 125 (2001).

[28] S.T. Hong, W.T. Kim, Y.J. Park, and M.S. Yoon, Phys. Rev. D 62, 085010 (2000).

[29] R. Zucchini, Int. J. Geom. Meth. Mod. Phys. 1, 813 (2004).

[30] K. Kondo, Phys. Lett. B 600, 287 (2004); Int. J. Mod. Phys. A 20, 4609 (2005); K. Kondo, T. Murakami, and T. Shinohara, Euro Phys. J. C 42, 475 (2005); S. Kato et al, Phys. Lett. B 71, 133 (2006).

[31] W.T. Kim, Y.W. Kim, M.I. Park, Y.J. Park, and S.J. Yoon, J. Phys. G 23, 325 (1997); Y.W. Kim and K. D. Rothe, Nucl. Phys. B 510, 511 (1998).

[32] L.D. Faddeev, Theor. Math. Phys. 1, 1 (1970); P. Senjanovich, Ann. Phys. 100, 277 (1976). 
[33] E.S. Fradkin, G.A. Vilkovisky, Phys. Lett. B 55, 224 (1975); M. Henneaux, Phys. Rep. 126, 1 (1985).

[34] T. Fujiwara, Y. Igarashi and J. Kubo, Nucl. Phys. B 341, 695 (1990); Y.W. Kim, S.K. Kim, W. T. Kim, Y.J. Park, K. Y. Kim, and Y. Kim, Phys. Rev. D 46, 4574 (1992); R. Banerjee, H.J. Rothe, and K.D. Rothe, Phys. Rev. D 49, 5438 (1994); C. Bizdadea and S.O. Saliu, Nucl. Phys. B 456, 473 (1995). 